\title{
Phonological and visual working memory in mental addition
}

\author{
PATRICIA L. TRBOVICH and JO-ANNE LEFEVRE \\ Carleton University, Ottawa, Ontario, Canada
}

\begin{abstract}
The goal of the present research was to examine the role of working memory in mental arithmetic. Adults $(n=96)$ solved multidigit arithmetic problems (e.g., $52+3 ; 3+52)$ alone and in combination with either a phonological memory load (i.e., nonwords, such as gup) or a visual memory load (i.e., random pattern of asterisks). The participants solved problems presented in a vertical format significantly faster than problems presented in a horizontal format. They also solved double digit first problems (e.g., $52+3)$ more quickly than the reverse (e.g., $3+52$ ), but only when the problems were presented horizontally. Performance was worse in the phonological load condition than in the visual load condition for the participants who solved problems presented horizontally, whereas performance was worse in the visual load condition than in the phonological load condition when problems were presented vertically. The present research provides evidence that both phonological and visual aspects of working memory are involved in mental arithmetic but that the role of each working memory component will depend on such factors as presentation format.
\end{abstract}

Solving an addition problem, such as $37+6$, involves a variety of mental activities. These activities may include accessing a memory representation (e.g., $7+6=$ 13), retention of the intermediate sum (e.g., 13), incrementing the decade $(30+10=40)$, assembly of an answer (e.g., $40+3$ ), and generation of a phonological code (e.g., /forti- $\theta$ ri/; Widaman, Geary, Cormier, \& Little, 1989). Given the multistep nature of mental arithmetic and the need for coordination of the various processes, it seems plausible that working memory is involved in these solutions (DeStefano \& LeFevre, in press). Working memory refers to the temporary storage and processing of information during complex cognitive activities (Miyake $\&$ Shah, 1999). Although there are a variety of models of working memory, Baddeley's (1986; Baddeley \& Hitch, 1974; Baddeley \& Logie, 1999) multicomponent model has most often been used to explore the role of working memory in mental arithmetic (DeStefano \& LeFevre, in press). The goal of the present research was to explore the working memory processes involved in addition problems, such as $43+5$ and $6+85$, within the multicomponent framework.

This experiment was part of P.L.T's thesis, conducted in partial fulfillment of the requirements for the M.A. degree at the Department of Psychology, Carleton University. We gratefully acknowledge the contributions of Michael Fox, Joanne Harbluk, Chris Herdman, and William Petrusic as members of the thesis committee and for their valuable insights. This research was supported by a research grant from the Natural Sciences and Engineering Research Council of Canada to J.-A.L. We thank Ben Coleman, Diana DeStefano, Marcie PennerWilger, and Brenda Smith-Chant for their helpful comments on earlier versions of this article. Correspondence concerning this article should be addressed to P. L. Trbovich, Department of Psychology, Carleton University, Ottawa, ON, K1S 5B6Canada (e-mail: ptrbovich@ @ rogers.com or jo-anne_lefevre@carleton.ca).
The multicomponent model of working memory comprises a limited-capacity central executive, a phonological loop, and a visual-spatial sketchpad (VSSP; Baddeley, 1986, 1996; Baddeley \& Hitch, 1974; Baddeley \& Logie, 1999). The central executive coordinates the actions of the phonological loop and the VSSP and is associated with limitations in the availability of cognitive resources (Baddeley, 1996). The phonological loop is involved in the temporary storage of verbal information (Baddeley, 1986), whereas the VSSP functions as a mental blackboard or workspace for temporary storage of visual and spatial information (Logie, 1995). The research on working memory and arithmetic reviewed by DeStefano and LeFevre (in press) indicated that all arithmetic problems involve central executive resources, even singledigit problems such as $3+2$. There was little evidence that the memory subsystems were involved in singledigit solutions, at least when the problems were solved via retrieval of the answer from memory (e.g., Hecht, 2002). For multidigit problems, however, research indicated that both the phonological loop and the VSSP may have a role in solving the problems, although there was no consensus on the conditions under which each memory subsystem is activated. In the present research, we used a memory load paradigm to explore the roles of the phonological loop and the VSSP in single- + double-digit and double- + single-digit addition problems.

In mental addition, the role of the phonological loop may be to retain information in a verbal form during ongoing calculations (DeStefano \& LeFevre, in press). Evidence for such a role was found when participants solved multidigit problems such as $395+256$ or $18 \times 9$ (Ashcraft \& Kirk, 2001; Fürst \& Hitch, 2000; Logie, Gilhooly, \& Wynn, 1994; Seitz \& Schumann-Hengsteler, 
2000, 2002), whereas there was little evidence for phonological coding when participants solved singledigit problems such as $4+9$ (De Rammelaere, Stuyven, \& Vandierendonck, 1999, 2001; Lemaire, Abdi, \& Fayol, 1996; Seitz \& Schumann-Hengsteler, 2000). The phonological load associated with multidigit problems increased when the operands were displayed for a limited time and as the complexity of the calculations increased (Fürst \& Hitch, 2000; Heathcote, 1994). Such results suggested that phonological codes might be used to retain operands or intermediate sums during solution (DeStefano \& LeFevre, in press). In the present experiment, we hypothesized that phonological codes would be involved in retention of intermediate sums, because the problems were shown continuously until the participant responded and, thus, there was no need for the participants to retain the operands.

Hitch (1978) and Hayes (1973) also suggested that both problem operands and intermediate results are held in working memory. They argued that this temporary storage was done in the VSSP, however (see also Heathcote, 1994). For example, Hayes suggested that the visual imagery used in mental arithmetic acts as a substitute for the notations (e.g., intermediate sums or carries) that would be used on paper during the performance of written arithmetic. This proposal seems counter to the research discussed above, in which verbal codes were found to be involved in the retention of intermediate sums. We hypothesized that the recruitment of phonological versus visual-spatial codes depends on problem format, as will be described below. Central to this assumption are two related findings: (1) Adults have access to a variety of solution procedures when they solve arithmetic problems (Hecht, 1999, 2002; LeFevre, Sadesky, \& Bisanz, 1996; Seyler, Kirk, \& Ashcraft, in press), and (2) processing of arithmetic problems varies with presentation format (e.g., Campbell, 1994; LeFevre, Lei, Smith-Chant, \& Mullins, 2001; Noël, Fias, \& Brysbaert, 1997; Noël \& Seron, 1997).

In the present research, we presented the arithmetic problems in two formats (see Figure 1). Half of the participants saw the operands in the problem presented horizontally, and the other participants saw the operands aligned vertically. We hypothesized that the participants would solve problems presented in vertical format by using the same unit-to-decade addition algorithm that they use on paper. Thus, in accord with Hitch (1978) and Hayes (1973), we hypothesized that the vertical format would activate temporary representations in the VSSP, leading to a greater involvement of VSSP codes in vertical than in horizontal presentation. Because recent research has indicated that visual and spatial codes are separable in working memory and because the visualization aspect was central to our hypothesis, in the present research we focused on the role of visual, rather than spatial, codes during arithmetic solutions (Coleman \& LeFevre, 2002; Della Sala, Gray, Baddeley, Allamano, \& Wilson, 1999; Logie \& Marchetti, 1991).

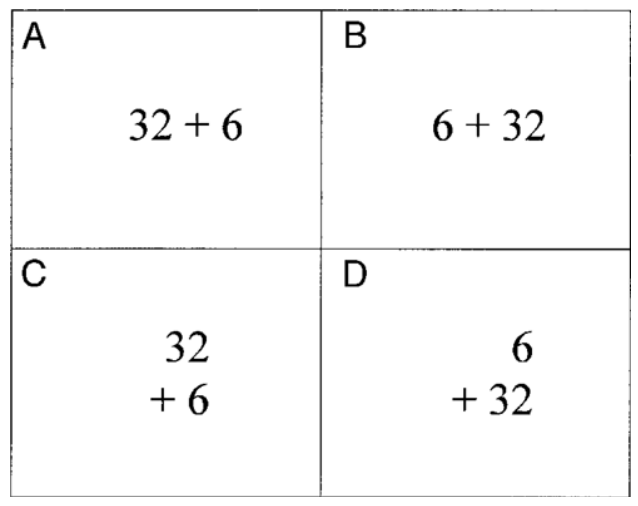

Figure 1. Problem formats: (A) double digit first, horizontal, (B) single digit first, horizontal, (C) double digit first, vertical, and (D) single digit first, vertical.

In contrast, the participants were expected to rely on a variety of solution approaches when the problems were presented in horizontal format. For example, they might solve a problem such as $79+4$ by recomposing it as 80 $+(4-1)$. Such alternatives to the standard algorithm may be more likely to activate phonological codes if the revised operands and intermediate sums are held in the phonological loop. Thus, we predicted that horizontally presented problems would be more likely to activate phonological codes than vertically presented problems would be. In horizontal presentation, another aspect of problem format was also expected to play a role. Brysbaert, Fias, and Noël (1998) found that the order of the operands in horizontally presented problems (e.g., $4+31$ vs. $31+4)$ influenced solution times when participants had to produce a verbal answer. For French-speaking participants, $31+4$ was solved more quickly than $4+31$, whereas Dutch-speaking participants showed the opposite pattern. Note that the answer 31 is named as the equivalent of "one-and-thirty" in Dutch versus "thirtyand-one" in French. If the participants were activating verbal codes for each operand, one explanation for the operand order $\times$ language interaction is that the compatibility between the positions of the decade digit in the input form and in the spoken output facilitates construction of the answer. In support of this view, Brysbaert et al. found that the pattern of facilitation shown by the Dutch-speaking participants reversed (i.e., became the same as that shown by the French speakers) when the answer had to be typed. In contrast to the effects of operand order in horizontal problems, operand order is not expected to influence the processing of vertical problems. If the operand order effect reflects interactions between phonological processing at calculation and answer production, observing such an effect in horizontal problems provides additional support for the prediction that phonological codes would be more highly activated in horizontal than in vertical presentation.

In the present experiment, a memory load paradigm was used to explore the involvement of the phonological 
loop and the VSSP in mental addition. Participants solved arithmetic problems while they retained either a phonological load (i.e., consonant-vowel-consonant [CVC] nonwords such as gup) or a visual load (i.e., a pattern of asterisks). Observation of mutual interference between the arithmetic task and the memory load task is assumed to indicate that the two tasks use the same processing resources or processing codes. The visual load (i.e., a static pattern) was similar to the stimuli used, for example, in the visual pattern span task (Della Sala, Gray, Baddeley, \& Wilson, 1997). Performance on similar visual patterns is more affected when combined with visual interference than when combined with spatial interference (e.g., Coleman \& LeFevre, 2002; Della Sala et al., 1999). The memory load paradigm has been used in a similar way to explore the role of different mental codes in a variety of complex cognitive tasks (Ashcraft \& Kirk, 2001; Herdman \& Beckett, 1996; Seyler et al., in press).

In summary, research on the relation between working memory and arithmetic suggests that both the phonological loop and the VSSP may be involved in addition performance. The participants in the present research solved single- plus double-digit addition problems. For half of the participants, the problems were presented horizontally (e.g., $7+65$ ). For the other participants, the problems were presented vertically, with the single digit aligned with the unit value of the double digit (see Figure 1). We hypothesized that horizontal problems would be solved more slowly than vertical problems (Heathcote, 1994). We also predicted that single- + double-digit problems would take longer to solve than double- + singledigit problems when the problems were presented horizontally (Brysbaert et al., 1998). To assess working memory demands, the participants retained a memory load while solving the problems. The difficulty of the memory load was varied. In the control condition, there was no memory requirement. The easy phonological load was one CVC nonword (e.g., gub), and the hard phonological load was three CVC nonwords. The easy visual load was a random pattern of four asterisks, and the hard visual load was a pattern of eight asterisks. We hypothesized that performance in the horizontal condition would be worse as phonological load increased. In contrast, we expected that performance in the vertical condition would be worse as visual load increased.

\section{METHOD}

\section{Participants}

Ninety-six participants (55 males and 41 females) received either course credit (as partial fulfillment of a course requirement) or $\$ 10$ for their participation. The participants ranged in age from 18 to 51 years, with a median of 19 .

\section{Design}

The participants solved 72 addition problems in one of four formats by memory load conditions. Forty-eight participants solved problems presented horizontally (see Figure 1), and 48 participants solved problems presented vertically. Half of the participants in each format condition were given a phonological memory load, and half were given a visual memory load (described in detail below). For each participant, 36 problems were presented with the single digit first (e.g., $4+57$ ), and half were presented with the double digit first (e.g., $57+4)$. One third of the problems were presented without a memory load (control), one third with an easy load, and one third with a hard load (load levels will be described in more detail below). Thus, the design was a 2 (format: horizontal or vertical) $\times 2$ (type of memory load: phonological or visual) $\times 2$ (operand order: single digit first vs. double digit first) $\times 3$ (level of load: control, easy, or hard) mixed factors design with repeated measures on the last two factors.

\section{Apparatus}

An IBM 80286 computer was used to present stimuli on a video monitor and to record responses. Response latencies were detected using a headset containing a built-in microphone. The participants were seated approximately $50 \mathrm{~cm}$ in front of the video monitor. Trials were self-paced, with the participants pushing the center key on a three-button response panel to start each trial. Memory task responses were made using the remaining two keys.

\section{Materials}

Thirty-six arithmetic problems with sums less than 100 were selected. Each problem was presented twice, once with the single digit first (e.g., $5+47$ ) and once with the double digit first (e.g., $47+$ $5)$. The single-digit components of the problems in each set consisted of the numbers from 3 to 8 . An equal number of problems had double-digit operands from the decades 30 through 80 . Each participant solved 12 problems in each of the six conditions formed by crossing load (i.e., control, easy, or hard) $\times$ operand order (single first vs. double first). Half of the problems in each condition involved carrying (e.g., $6+37$ ), and half did not (e.g., $6+83) .{ }^{1}$ The problems were grouped into three sets of 24 (with the aforementioned constraints), and combinations of set and levels of load were counterbalanced across participants. Two randomly ordered lists of each of the three combinations of sets and loads were generated, for a total of six different lists. Equal numbers of participants were assigned to each list across format and memory load conditions (i.e., 4 per condition).

Phonological memory task. The phonological memory items were pronounceable CVC nonwords (e.g., gub, nof, sul). The CVCs were constructed so that they did not spell a word, proper name, or common abbreviation. Separate lists of single CVCs $(n=34)$ and combinations of three CVCs $(n=34)$ were constructed. Each CVC was used only once during the experiment. Across participants, CVC items were selected randomly without replacement on each trial. Thus, each participant saw a different combination of problems and CVC items. On half of the easy load trials, the probe CVC (i.e., one that was to be compared with the initial memory load) matched the initial CVC. On half of the hard load trials, the probe CVC matched one of the three CVCs in the initial set. On nonmatching trials, probe CVCs differed from initial CVCs by only a single letter. For example, for the memory load gub nof sul the nonmatching probe item was gup. Approximately the same numbers of nonmatching CVCs from the hard load came from the first, second, and third positions.

Visual memory task. The visual memory loads were patterns of asterisks arranged in a $5 \times 5$ array. Grid lines were not visible. For the easy load, four asterisks were presented. For the hard load, eight asterisks were presented. The visual loads were selected so that they did not form recognizable objects or letters, to discourage the participants from using a verbal label as a memory aid. Lists of fourasterisk $(n=34)$ and eight-asterisk $(n=34)$ patterns were constructed. On each trial, a pattern was selected randomly without replacement from the list of patterns for that level of load. Nonmatching patterns were constructed by moving one of the asterisks 
by a single position in the array (up, down, left, or right). Half of the probe patterns at each level of load matched the initial pattern.

Other measures. The participants completed a paper-and-pencil arithmetic fluency test that included multidigit addition, subtraction, and multiplication problems (French, Ekstrom, \& Price, 1963). Numbers of correct problems on each page of the addition and subtractionmultiplication tests were summed to yield a total fluency score. The participants also completed the Math Background and Interests Questionnaire, which includes a series of questions about their educational background and experiences and their attitudes and beliefs about mathematics (see LeFevre, Smith-Chant, Hiscock, Daley, \& Morris, 2003, for detailed information about this survey).

\section{Procedure}

Half of the participants $(n=48)$ were randomly assigned to the phonological memory task, and half $(n=48)$ were randomly assigned to the visual memory task. Every participant first completed 20 practice trials. Trial-by-trial feedback on both the arithmetic and the memory tasks was given in the practice block. Each trial began with the word READY shown in the center of the screen. READY was displayed for $1 \mathrm{sec}$, then flashed on and off twice at 500-msec intervals. On what would have been the fourth appearance of READY, the load (phonological or visual, as will be described below) appeared. The load was displayed for $1,500 \mathrm{msec}$, and then the screen was blank for $1 \mathrm{sec}$ to allow the participant time to rehearse the load. Next, the arithmetic problem appeared and remained on the screen until the participant responded or for a maximum of $10 \mathrm{sec}$. The screen was blank for $1 \mathrm{sec}$, and then the probe appeared. The probe remained on the screen until the participant responded or until $20 \mathrm{sec}$ had elapsed. The experimenter recorded the participant's response to the arithmetic problem during this interval. No feedback was given on experimental trials. After the participant had responded to the probe by pressing a button on the response panel, the screen was blank for $2 \mathrm{sec}$. The participants were asked to respond as quickly and accurately as possible to both tasks.

Phonological memory task. The participants saw three kinds of memory loads: a single asterisk in the center of the screen, one nonword in the center of the screen, or three nonwords (arranged horizontally) in the center of the screen. They were instructed to subvocally rehearse the nonwords and to place the same emphasis on both tasks. In the control condition, a single asterisk appeared both as the memory load and as the probe, and the participants simply pressed the response button to continue. In the easy (one CVC) and hard (three CVCs) load conditions, the probe was always a single CVC. The participants indicated whether the probe was the same as or different from the memory load (or matched one of the three initial CVCs) by pressing the appropriate key on a three-key response panel.

Visual memory task. On each trial, the participants saw one of three types of memory patterns in the center of the screen: a single asterisk, a pattern of four asterisks, or a pattern of eight asterisks. The participants were encouraged to keep the asterisk pattern in memory while solving the addition problem and to place the same emphasis on both tasks. In the control condition, a single asterisk appeared both as the memory load and as the probe pattern, and the participants simply pressed the response button to continue. The probe pattern always had the same number of asterisks as the initial memory load. In the four- and eight-asterisk conditions, the participants indicated whether the second pattern of asterisks was the same as or different from the first pattern by pressing the appropriate key on a three-key response panel. The participants were encouraged to keep the asterisk pattern in memory while solving the addition problem and to place the same emphasis on both tasks.

Other measures. The participants were given 2 min per page on the arithmetic fluency tests and were instructed to complete as many problems as possible. After finishing all the other tasks, the participants spent approximately 5 min completing the Math Background and Interests Survey.

\section{RESULTS}

\section{Arithmetic Fluency}

The participants' mean scores on the arithmetic fluency test were used to determine whether the groups were equivalent in overall computational skill. Fluency score was the total number of problems solved correctly across the four pages of this test (maximum 240). Scores on the arithmetic fluency test were analyzed in a 2 (memory task: visual vs. phonological) $\times 2$ (format: vertical vs. horizontal) between-subjects analysis of variance (ANOVA). No main effects or interactions were significant, suggesting that the four groups were reasonably similar in arithmetic fluency. Although the groups did not vary in fluency, the mean of 71 was lower than the expected mean of 80 for this population $[t(95)=30.59, p<.001$; LeFevre et al., 2003]. Overall, about $75 \%$ of the participants in the present experiment were average or below average in arithmetic fluency, as indexed by this test.

\section{Dual-Task Performance}

The participants completed a total of 6,912 trials. They made nonanswer vocalizations or responded too quietly on $2 \%$ of the trials and made arithmetic errors on $8 \%$ of trials. Two indices of performance were calculated and analyzed: latencies to arithmetic problems and a combined error score. Only trials for which responses to both the arithmetic problem and the secondary task were correct were included in the analyses of latencies $(n=$ $5,407)$. The combined error score was the percentage of trials in each condition on which the participants made an error on either the arithmetic task or the memory task (or both). The assumption is that a breakdown in performance on either the arithmetic or the memory load task indexed working memory demands (Ashcraft \& Kirk, 2001; Seyler et al., in press). The combined error scores and the median correct latencies to arithmetic problems were analyzed in separate 2 (memory task: visual vs. phonological) $\times 2$ (format: vertical vs. horizontal) $\times 2$ (operand order: single digit + double digit vs. double digit + single digit $) \times 3$ (load: control, easy, or hard) ANOVAs, with repeated measures on the last two factors. Unless otherwise indicated, the alpha level was .05. Comparisons between means were made using $95 \%$ confidence intervals (calculated using the formula recommended by Loftus \& Masson, 1994). The mean latencies, standard deviations, and combined error scores in each condition are shown in the Appendix.

As was predicted, the participants responded more rapidly when the problems were presented in a vertical format than when the problems were presented horizontally $\left[1,556\right.$ vs. $\left.1,829 \mathrm{msec} ; F(1,92)=9.53, M S_{\mathrm{e}}=1,128,123\right]$. Also as was predicted, the participants solved double- + single-digit problems (e.g., $43+9 ; M=1,642 \mathrm{msec}$ ) more quickly than single- + double-digit problems [e.g., $9+43$; $\left.M=1,743 \mathrm{msec} ; F(1,92)=27 \cdot 23, M S_{\mathrm{e}}=53,839\right]$. Furthermore, operand order interacted with format $[F(1,92)=$ $\left.19.46, M S_{\mathrm{e}}=53,839, C I=47 \mathrm{msec}\right]$. The participants solved double- + single-digit problems significantly more 
quickly than single- + double-digit problems in the horizontal format (1,736 vs. $1,922 \mathrm{msec})$, whereas there was no difference in latencies across operand order in the vertical format (1,563 vs. $1,548 \mathrm{msec})$. These results replicate the pattern observed by Brysbaert et al. (1998) for horizontally presented problems.

Latencies did not differ significantly in phonological versus visual load conditions $[1,762$ vs. $1,623 \mathrm{msec}$; $\left.F(1,92)=2.52, M S_{\mathrm{e}}=1,128,123\right]$, and the main effect of level of load was not significant $(F<1)$. The interaction between load type (phonological vs. visual) and level of load was significant, however $[F(2,184)=3.73]$, as was the three-way interaction of these variables with format $\left[F(2,184)=4.10, M S_{\mathrm{e}}=62,657, C I=50 \mathrm{msec}\right]$. As is shown in Figure 2, performance on the arithmetic problems varied substantially with the type of load (phonological vs. visual) and format (horizontal vs. vertical). Interpretation of these patterns, however, is best done simultaneously with the results for the combined error score.

Analysis of the combined error score indicated that although the absolute levels of errors did not differ for phonological and visual loads $(F<1)$, format and type of load interacted $\left[F(1,92)=13.91, M S_{\mathrm{e}}=444.1\right]$. The participants who solved horizontally presented problems made more errors under phonological load than under visual load ( $20 \%$ vs. $15 \%)$, whereas the participants who solved vertically presented problems made more errors under visual load than under phonological load (22\% vs. $14 \%)$. These tradeoffs suggest differential involvement of phonological and visual working memory as a function of problem format. Errors increased with level of load $\left[F(2,184)=110.14, M S_{\mathrm{e}}=167.5, C I=2.6 \% ; 8 \%\right.$ in the control condition, $18 \%$ in the easy load, and $27 \%$ in the hard load]. Consistent with the results for latencies, the three-way interaction of type of load, format, and level of load was significant for errors $[F(2,184)=$ $\left.13.10, M S_{\mathrm{e}}=167.5, C I=2.6 \%\right]$, as is shown in Figure 2 (bottom half of each panel). There were no effects of operand order and no interactions with operand order in the analysis of errors.

Consider the results for the participants given a phonological load (top graph in Figure 2). Under either easy or hard phonological load, the participants who solved horizontally presented problems made significantly more errors than did participants who solved problems presented vertically. Latencies on arithmetic problems increased with load in the horizontal format, so that latencies were significantly longer in the hard condition than in the easy or the control condition. This pattern of performance for latencies and errors suggests that the problems that were presented horizontally involved phonological codes more than did the problems that were presented vertically. For problems presented vertically, latencies and errors increased from the control to the easy load condition, suggesting that the phonological load did interfere somewhat with performance. The subsequent decline in latencies for vertical problems in the hard load condition may represent a
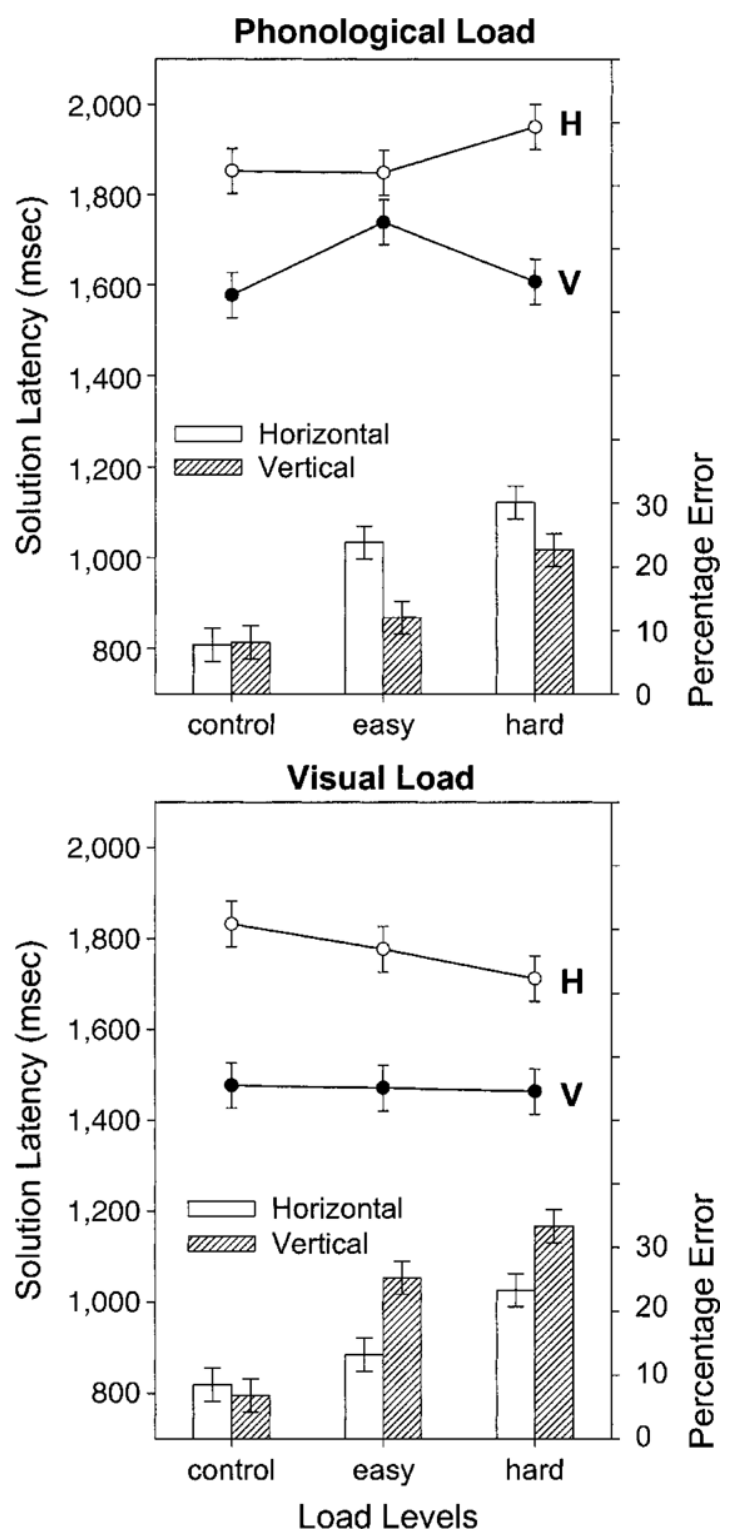

Figure 2. Solution latencies (in milliseconds) to arithmetic problems as a function of memory task, level of load, and presentation format are shown on the top of each panel. Combined error percentages are shown in the bottom part of each panel. For both measures, error bars represent $95 \%$ confidence intervals, based on the $M S_{\mathrm{e}}$ from the three-way interaction.

speed-accuracy tradeoff: Although the participants made fewer errors in the vertical condition than in the horizontal condition under a hard load, the difference between the vertical and the horizontal conditions was smaller than that in the easy load condition. In summary, although the phonological load influenced performance on both the horizontal and the vertical problems, the extent of interference between tasks was greater when the participants solved horizontal problems.

Next, consider the results for the participants given a visual load (bottom graph in Figure 2). These results 
show patterns that are opposite to those for the phonological load. In both the easy and the hard load conditions, the participants made more errors when the problems were presented vertically than when they were presented horizontally. Latencies on vertically presented problems did not vary with load, and latencies on horizontally presented problems decreased with load, suggesting that the participants protected arithmetic performance at the cost of errors. The decline in latencies with load in the horizontal condition was also observed by Kulak (1993). Because errors increased with load in this condition, however, the results appear to reflect a tradeoff between latencies and error scores. In summary, the vertical problems required more visual than phonological memory resources. These effects of visual load occurred mainly in patterns of errors across trials, not on latencies to arithmetic problems. Thus, allocation of resources to arithmetic versus memory task performance depended on the type of memory load required. These findings suggest that it is important to evaluate both arithmetic and memory load performance when interpreting the results of dual-task experiments.

\section{DISCUSSION}

Researchers have suggested that both the phonological and the visual-spatial subsystems of working memory may be involved in the solution of arithmetic problems (Fürst \& Hitch, 2000; Hayes, 1973; Heathcote, 1994; Hitch, 1978; Hunter, 1979; Lee \& Kang, 2002; Lemaire et al., 1996; Logie et al., 1994; Noël, Désert, Aubrun, \& Seron, 2001; Zago et al., 2001). The results of the present research support this view and provide detailed information about the conditions that influence the recruitment of phonological versus visual codes.

First, the problems presented in vertical format required more visual resources than did the problems presented in horizontal format. These findings are consistent with the view that the vertical format activates a representation of the problem in the VSSP in a form that is similar to that used when people solve paper-and-pencil addition problems. The alignment of the unit digits obviated the effect of operand order found for horizontal presentation (Brysbaert et al., 1998) and resulted in overall shorter latencies (Heathcote, 1994). Because the effects of visual load occurred mainly in the errors, whereas latencies on the arithmetic problems were protected, one interpretation is that the vertical presentation stabilized performance by imposing a consistent solution procedure on the participants. The increased incidence of errors on the visual memory task in the vertical condition, relative to the horizontal condition, however, shows that there are visual resources associated with the use of this solution procedure.

Second, the problems presented in horizontal format required more phonological resources than did the problems presented in vertical format. The requirement for phonological resources may be related to participants' selection of solution procedures in horizontal versus ver- tical presentation. Participants clearly find horizontal problems more difficult (independent of load) than vertical problems. This disadvantage is tempered somewhat when problems are presented with the double digit first (e.g., $34+3)$. It may be that this operand order allows the unit digits to be aligned more easily than when the single digit occurs first (e.g., $3+34$ ). Participants may also be more likely to use procedures that require multiple steps and, thus, retention of more intermediate sums (e.g., converting problems such as $7+38$ to $40+7-2$ ) when the problems are presented horizontally than when they are presented vertically. Hecht (2002) found that the working memory demands of solving single-digit problems were greater when participants used such multiplestep procedures, with phonological codes especially implicated in solutions that involved counting (see also Seyler et al., in press). In summary, the differential recruitment of phonological versus visual memory in horizontal and vertical problems may be related to variability in the solution procedures that participants select as a function of format.

The present findings provide an important perspective on the role of the working memory subsystems in mental arithmetic. Problems presented horizontally seemed to implicate phonological codes, whereas problems presented vertically appeared to activate visual codes. The importance of presentation manipulations (vertical vs. horizontal and operand order) in interpreting the present results suggests that researchers in the area of mathematical cognition should routinely manipulate these factors. Comparing across experiments that use different presentation conditions is likely to result in apparent inconsistencies and contributes to a lack of coherence in the literature (DeStefano \& LeFevre, in press). Furthermore, the complexity of the problems (i.e., number of digits in each operand, number of steps required for solution) used in any given experiment is likely to influence the relations between experimental manipulations and performance. In summary, the present results support the view that working memory is very important in mental arithmetic (Ashcraft, Donley, Halas, \& Vakali, 1992; Ashcraft \& Kirk, 2001) and provide some much needed detail about how specific mental codes and processes are involved in the solution of addition problems.

\section{REFERENCES}

Ashcr aft , M. H., Donl ey, R. D., Hal as, M. A., \& Vakal i, M. (1992). Working memory, automaticity, and problem difficulty. In J. I. D. Campbell (Ed.), The nature and origins of mathematical skills (pp. 301-329). Amsterdam: Elsevier, North-Holland.

Ash cr aft , M. H., \& Kir k, E. P. (2001). The relationships among working memory, math anxiety, and performance. Journal of Experimental Psychology: General, 130, 224-237.

Baddel ey, A. D. (1986). Working memory. Oxford: Oxford University Press.

Baddel ey, A. D. (1996). Exploring the central executive. Quarterly Journal of Experimental Psychology, 49A, 5-28.

Baddel ey, A. D., \& Hit ch, G. J. (1974). Working memory. In G. H. Bower (Ed.), The psychologyoflearning and motivation: Advances in research and theory (Vol. 8, pp. 47-89). New York: Academic Press. Baddel ey, A. D., \& Logie, R. H. (1999). Working memory: The 
multiple-component model. In A. Miyake \& P. Shah (Eds.), Models of working memory: Mechanisms of active maintenance and executive control (pp. 28-61). New York: Cambridge University Press.

Brysbaert, M., Fias, W., \& Noël, M.-P. (1998). The Whorfian hypothesis and numerical cognition: Is 'twenty-four' processed in the same way as 'four-and-twenty'? Cognition, 66, 51-77.

Campbel1, J. I. D. (1994). Architectures for numerical cognition. Cognition, 53, 1-44.

Col eman, B., \& LeFevre, J.-A. (2002, May). Fractioning the visuospatial sketchpad: Active processing required! Paper presented at the annual meeting of the Canadian Society for Brain, Behaviour, \& Cognitive Science, Vancouver.

Del 1 a Sal a, S., Gray, C., Baddel ey, A. D., All a mano, N., \& Wil son, L. (1999). Pattern span: A tool for unwelding visuo-spatial memory. Neuropsychologia, 37, 1189-1199.

Del 1 a Sal a, S., Gray, C., Baddel ey, A. D., \& Wil son, L. (1997). Visual patterns test: A test of short-term visual recall. London: Thames Valley Test Company.

De Rammel aere, S., St uyven, E., \& Vandierendonck, A. (1999). The contribution of working memory resources in the verification of simple arithmetic sums. Psychological Research, 62, 72-77.

De Rammel aere, S., St uyven, E., \& Vandier endonck, A. (2001). Verifying simple arithmetic sums and products: Are the phonological loop and the central executive involved? Memory \& Cognition, 29, 267-273.

DeSt ef a no, D., \& LeFevre, J.-A. (in press). The role of working memory in mental arithmetic. European Journal of Cognitive Psychology.

French, J. W., Ekst rom, R. B., \& Price, I. A. (1963). Kit of reference tests for cognitive factors. Princeton, NJ: Educational Testing Service.

Fürst, A. J., \& Hit ch, G. J. (2000). Separate roles for executive and phonological components of working memory in mental arithmetic. Memory \& Cognition, 28, 774-782.

Hayes, J. R. (1973). On the function of visual imagery in elementary mathematics. In W. Chase (Ed.), Visual information processing (pp. 177-214). New York: Academic Press.

Heat hcote, D. (1994). The role of visuo-spatial working memory in the mental addition of multi-digit addends. Current Psychology of Cognition, 13, 207-245.

Hech t, S. A. (1999). Individual solution processes while solving addition and multiplication math facts in adults. Memory \& Cognition, 27, 1097-1107.

Hech t, S. A. (2002). Counting on working memory in simple arithmetic when counting is used for problem solving. Memory \& Cognition, 30, 447-455.

Her dma n, C. M., \& Becket t, B. L. (1996). Code-specific processes in word naming: Evidence supporting a dual-route model of word recognition. Journal of Experimental Psychology: Human Perception \& Performance, 22, 1149-1165.

Hit ch, G. J. (1978). The role of short-term working memory in mental arithmetic. Cognitive Psychology, 10, 203-323.

Hunt er, J. M. L. (1979). Memory in everyday life. In M. M. Gruneberg \& P. E. Morris (Eds.), Applied problems in memory (pp. 1-23). New York: Academic Press.

Kul ak, A. G. (1993). Spatial processing and arithmetic performance. Unpublished $\mathrm{PhD}$ thesis, Carleton University.

Lee, K., \& Kang, S. (2002). Arithmetic operation and working memory: Differential suppression in dual tasks. Cognition, 83, B63-B68.

LeFevre, J.-A., Lei, Q., Smith-Chant, B., \& Mul1 ins, D. (2001). Multiplication by eye and by ear for Chinese-speaking and Englishspeaking adults. Canadian Journal of Experimental Psychology, 55, 277-284.

LeFevr e, J.-A., Sadesky, G. S., \& Bisan z, J. (1996). Selection of procedures in mental addition: Reassessing the problem-size effect in adults. Journal of Experimental Psychology: Learning, Memory, \& Cognition, 22, 216-230.

LeFevre, J.-A., Smit h-Chant, B. L., Hiscock, K., Dal ey, K. E., \& Morr is, J. (2003). Young adults' strategic choices in simple arith- metic: Implications for the development of mathematical representations. In A. J. Baroody \& A. Dowker (Eds.), The development of arithmetic concepts and skills: Constructing adaptive expertise (pp. 203-228). Mahwah, NJ: Erlbaum.

Lema ir e, P., Abdi, H., \& Fayol, M. (1996). The role of working memory resources in simple cognitive arithmetic. European Journal of Cognitive Psychology, 8, 73-103.

Loft u s, G. R., \& Masson, M. E. J. (1994). Using confidence intervals in within-subject designs. Psychonomic Bulletin \& Review, 1, 476490.

Logie, R. H. (1995). Visuo-spatialworking memory. Hillsdale, NJ: Erlbaum.

Logie, R. H., Gil hooly, K. J., \& Wynn, V. (1994). Counting on working memory in arithmetic problem solving. Memory \& Cognition, 22, 395-410.

Logie, R. H., \& Mar chet t i, C. (1991). Visuo-spatial working memory: Visual, spatial, or central executive? In R. H. Logie \& M. Denis (Eds). Mental images in human cognition (pp. 105-115). Amsterdam: North-Holland.

Miyake, A., \& Shah, P. (1999). Models of working memory: Mechanisms of active maintenance and executive control. Cambridge: Cambridge University Press.

Noël, M.-P., Déser t, M., Au br un, A., \& Ser on, X. (2001). Involvement of short-term memory in complex mental calculation. Memory \& Cognition, 29, 34-42.

Noël, M.-P., Fias, W., \& Brysbaert, M. (1997). About the influence of the presentation format on arithmetical-fact retrieval processes. Cognition, 63, 335-374.

Noël, M.-P., \& Ser on, X. (1997). On the existence of intermediate representations in numerical processing. Journal of Experimental Psychology: Learning, Memory, \& Cognition, 23, 697-720.

Seit z, K., \& Schumann-Hengst el er, R. (2000). Mental multiplication and working memory. European Journal of Cognitive Psychology, 12, 552-570.

Seit z, K., \& Schu mann-Hen gst el er, R. (2002). Phonological loop and central executive processes in mental addition and multiplication. Psychologische Beiträge, 44, 275-302.

Seyler, D. J., Kir k, E. P., \& Ashcr aft, M. H. (in press). Elementary subtraction. Journal of Experimental Psychology: Learning, Memory, \& Cognition.

Widaman, K. F., Gear y, D. C., Cor mier, P., \& Lit t 1 e, T. D. (1989). A componential model for mental addition. Journal of Experimental Psychology: Learning, Memory, \& Cognition, 15, 898-919.

Zago, L., Pesent i, M., Mell let, E., Crivel1 o, F., Mazoyer, B., \& Tzour io-Mazoyer, N. (2001). Neural correlations of simple and complex mental calculation. NeuroImage, 13, 314-327.

\section{NOTE}

1. We included a problem complexity manipulation in this experiment (i.e., carry vs. no-carry problems, such as $25+4$ vs. $28+4$ ) because our original intent was to evaluate working memory demands relative to this variable, in addition to the other factors. However, because errors across trials were relatively high and inclusion of the problem complexity variable resulted in a maximum of six items per condition, some means were based on only a few data points. This situation resulted in an unacceptable loss of power and complicated the interpretation of the effects that were significant. The participants showed the expected main effect of carry versus no-carry problems when latencies and errors were analyzed with this variable included. However, problem complexity did not interact with type of load for either latencies or errors and, thus, did not contribute to understanding the role of working memory in mental arithmetic. The lack of an interaction between load type (or level of load) and problem complexity has also been reported by a number of other researchers (see DeStefano \& LeFevre, in press, for details). Thus, in the present paper, we report data collapsed across problem complexity. 


\section{APPENDIX}

Mean Solution Latencies ( $M s$ ) and Standard Deviations ( $S D$ s; in Milliseconds) on Arithmetic Problems (in Milliseconds) and Percentage of Errors (Combined Across Arithmetic and Memory Load Tasks) as a Function of Memory Task (Phonological or Visual), Format (Horizontal or Vertical), Operand Order (Single Digit First vs. Double Digit First), and Level of Load (Control, Easy, or Hard)

\begin{tabular}{|c|c|c|c|c|c|c|c|c|c|}
\hline \multirow[b]{3}{*}{ Format } & \multicolumn{9}{|c|}{ Level of Load } \\
\hline & \multicolumn{3}{|c|}{ Control } & \multicolumn{3}{|c|}{ Easy } & \multicolumn{3}{|c|}{ Hard } \\
\hline & $M$ & $S D$ & $\%$ & $M$ & $S D$ & $\%$ & $M$ & $S D$ & $\%$ \\
\hline \multicolumn{10}{|c|}{ Phonological Load - Single Digit + Double Digit } \\
\hline Horizontal & 1,984 & 472 & 8.8 & 1,935 & 489 & 24.7 & 2,000 & 569 & 29.9 \\
\hline Vertical & 1,604 & 522 & 8.0 & 1,744 & 462 & 11.5 & 1,629 & 473 & 21.9 \\
\hline \multicolumn{10}{|c|}{ Visual Load - Single Digit + Double Digit } \\
\hline Horizontal & 1,924 & 571 & 10.7 & 1,877 & 548 & 13.7 & 1,811 & 513 & 23.3 \\
\hline Vertical & 1,501 & 431 & 6.7 & 1,455 & 442 & 26.3 & 1,447 & 461 & 34.4 \\
\hline \multicolumn{10}{|c|}{ Phonological Load - Double Digit + Single Digit } \\
\hline Horizontal & 1,721 & 424 & 6.6 & 1,762 & 485 & 23.0 & 1,902 & 454 & 30.2 \\
\hline Vertical & 1,550 & 419 & 8.1 & 1,732 & 587 & 12.4 & 1,585 & 427 & 23.4 \\
\hline \multicolumn{10}{|c|}{ Visual Load - Double Digit + Single Digit } \\
\hline Horizontal & 1,739 & 511 & 6.2 & 1,677 & 480 & 12.6 & 1,613 & 501 & 23.3 \\
\hline Vertical & 1,452 & 506 & 7.0 & 1,488 & 472 & 24.2 & 1,480 & 521 & 32.3 \\
\hline
\end{tabular}

(Manuscript received March 27, 2002;

revision accepted for publication March 10, 2003.) 